\title{
Computed Tomography Perfusion Can Guide Endovascular Therapy in Bilateral Carotid Artery Dissection
}

\author{
$\underline{\text { Isuru Induruwa }}^{1}$, Chloe Bentham $^{1}$, Kayvan Khadjooi ${ }^{1}$, Nikhil Sharma ${ }^{2}$ \\ ${ }^{1}$ Addenbrooke's Hospital, Cambridge University Hospitals NHS Foundation Trust, Cambridge, UK \\ ${ }^{2}$ The National Hospital of Neurology, Queen Square and MRC Unit for Lifelong Health and Ageing at UCL, London, UK
}

Received: $12 / 09 / 2015$

Accepted: 16/11/2015

Published: 15/12/2015

How to cite this article: Induruwa I, Bentham C, Khadjooi K, Sharma N. Computed tomography perfusion can guide endovascular therapy in bilateral carotid arthery dissection. EJCRIM 2015;2:doi:10.12890/2015_000295

Conflicts of Interests: The authors declare that there are no competing interests.

This article is licensed under a Commons Attribution Non-Commercial 4.0 License

\section{INTRODUCTION}

Carotid artery dissection (CAD) is a major cause of stroke in those under age 45, accounting for around $20 \%$ of ischaemic events ${ }^{[1,2]}$. In the absence of known connective tissue disorders, most dissections are traumatic ${ }^{[2]}$. First-line management is comprised of antiplatelet or anticoagulation therapy, but many traumatic dissections progress despite this and carry the risk of long-term complications from embolism or stenosis[3].

We report a case of traumatic bilateral carotid dissection leading to progressive neurological symptoms and hypoperfusion on computed tomography perfusion (CTP), despite escalation in anticoagulation, which led to emergency carotid stenting.

\section{LEARNING POINTS}

- Carotid artery dissection should always be considered in young patients presenting with stroke.

- Most strokes are caused by emboli from the injured vessel but hypoperfusion, especially from bilateral dissections, can also cause stroke.

- Anticoagulation or antiplatelets are used as first-line therapy, though there are no randomised control trials to guide management.

- Failure of medical therapy can be common and endovascular therapy should be considered in these cases.

- Computed tomography perfusion (CTP) scanning can be useful because it highlights areas of ischaemic penumbra that may be salvageable through such intervention.

\section{KEYWORDS}

Stroke, carotid, carotid artery dissection, CT perfusion, stenting, endovascular.

\section{CASE REPORT}

A 24-year-old asthmatic female presented to the emergency department following a road traffic accident. She was the restrained driver of a vehicle travelling at about $40 \mathrm{mph}$, when her vehicle collided with another vehicle pulling out of a side road. She self-extracted from the vehicle, but paramedics noted transient ( $<5$ minutes) left-sided hemiparesis and dysarthria at the scene.

Her admission computed tomography angiography (CTA) noted a right-sided carotid artery dissection and the patient was started on dual antiplatelet therapy. She was discharged the next day with plans for a magnetic resonance imaging (MRI) head and magnetic resonance (MR) angiography in one week. 
Three days later, she presented to the emergency department with fluctuating left hemiparesis (MRC 4/5) and dysarthria (NIHSS 7). A further CTA showed progression to bilateral CAD. IV heparin was added to dual antiplatelet therapy, with aggressive fluid management, in order to maintain cerebrovascular perfusion.

Overnight she deteriorated further, developing worsening left hemiparesis (MRC 1/5), neglect and agitation (NIHSS increased to 12). The CTP scan (Fig. 1) revealed an increased mean transit time (MTT), demonstrating that the cerebral circulation was slowed, with corresponding reduced cerebral blood flow (CBF) to the areas supplied by both her dissected carotid arteries. Nevertheless, at this point, the cerebral blood volume (CBV) was still preserved through cerebral autoregulation mechanisms. These perfusion parameters illustrated bilateral ischaemic penumbra of the entire anterior circulation, which was still salvageable and could relieve the patient's neurological burden. There was, however, a small corresponding hypodensity, representing an established infarct in her right frontal lobe on the plain computed tomography (CT) images, and it was felt that IV thrombolysis would carry considerable risk of haemorrhage.

Therefore, the decision was made for radiologically guided stenting in order to reduce the hypoperfusion burden from her bilateral dissections. Five endovascular stents were placed (3 R-ICA \& 2 L-ICA) with near complete resolution of flow on angiography (Fig. 2). A small area of matched MTT, CBF and CBV in her right frontal lobe representing the established infarct remained on follow-up CTP, but her perfusion parameters had normalised after stenting (Fig. 1).
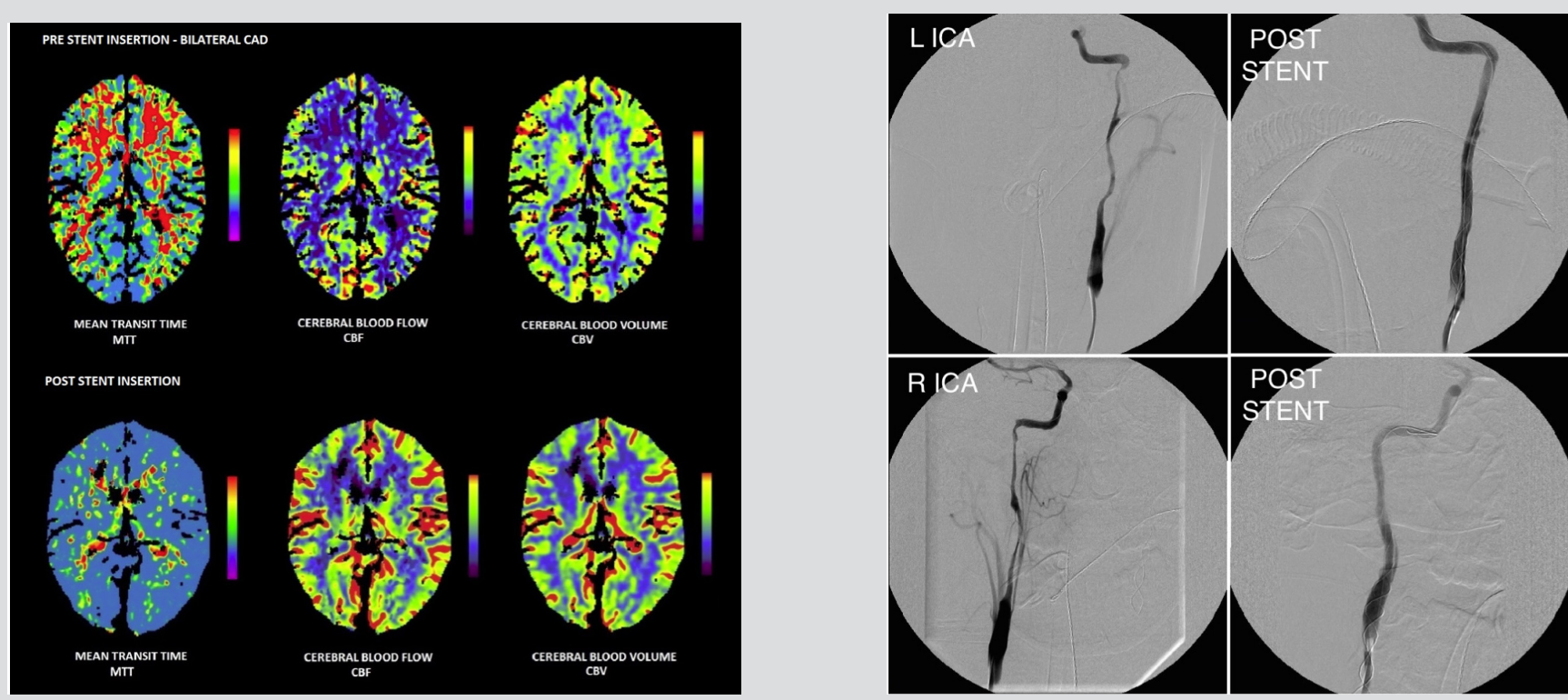

Figure 1. Computed tomography perfusion (CTP) scan showing improved perfusion parameters* post stent insertion.

Figure 2. Cerebral angiography of left and right ICA dissections and subsequent restoration of cerebral blood flow after successful stenting.

*Mean transit time (MTT): The average time (sec) required for blood to pass through tissue. In acute stroke, this time would be increased in order to maximise oxygenation of ischaemic tissue. The areas in red represent areas of increased MTT and, thus, the areas affected by bilateral CAD.

Cerebral blood flow (CBF): The flow rate of volume of blood through cerebral vasculature ( $\mathrm{ml} / 100 \mathrm{~g} / \mathrm{min})$. In acute stroke, this is reduced as a result of compromise to the blood supply. The areas in purple have the least cerebral blood flow and are at risk of stroke if cerebral autoregulation fails (red: blood vessels; green: normal cortex; blue: normal white matter; purple: low blood flow).

Cerebral blood volume (CBV): Defined as the volume of flowing blood (ml/100g). Cerebral autoregulation preserves this as long as possible in acute stroke in order to maintain cerebral perfusion (red: blood vessels; green: normal cortex; blue: normal white matter; purple: low blood volume).

Salvageable ischaemic penumbra is seen on CTP as increased MTT, reduced CBF but preserved CBV (as per the pre-stent images).

When MTT is increased and CBF and CBV are decreased; this is seen as the infarct core which is not salvageable. When an infarct matures, it is seen as 'black' on CTP. This is represented by the small right frontal infarct on post-stent images, which persisted, despite timely intervention. 


\section{DISCUSSION}

Most causes of CAD are due to underlying connective tissue disorders, such as Marfans or Ehlers-Danlos syndrome. However, $1 \%$ of dissections are traumatic, caused by either direct trauma, or the decelerating forces created during impact causing the neck to hyperextend. This causes intimal tearing and luminal compromise, with the subsequent blood flow and haematoma formation leading to dissection ${ }^{[2]}$. The management of CAD can be difficult and optimal therapy remains controversial due to the lack of randomised studies ${ }^{[4,5]}$. This is especially true of multiple and more occlusive dissections, where the clinical course can be slower and more unpredictable[6].

The use of anticoagulation stems from the understanding that micro-emboli from the dissected vessel most commonly lead to stroke $\mathrm{e}^{[7]}$. However, in this case, the fluctuating and progressive neurological burden was from worsening cerebral hypoperfusion due to critical stenosis of both carotid vessels. Despite early and careful escalation of anticoagulation, the patient's symptoms continued to worsen. Haemodynamically critical dissection and impending stroke is often seen as a reason for intervention in CAD ${ }^{[8]}$, and the use of CTP in these instances can guide management as it clearly highlights reduced perfusion parameters and any salvageable areas of penumbra. Furthermore, endovascular treatments have a high success rate in $C A D^{[9]}$, especially as anticoagulation does not always manage to negate the risk of stroke. Clinicians can be left in a difficult situation in stroke post CAD, as patients are often anticoagulated and interventions, such as IV thrombolysis, therefore carry a considerable risk of haemorrhage.

Although improvements both clinically and in CTP parameters have been seen after stenting in patients with high-grade carotid artery stenosis ${ }^{[10]}$, there is limited literature describing it in bilateral CAD. This case highlights how emergency bilateral stenting can be successful in selected cases of progressive dissection, guided by CTP.

\section{CONCLUSION}

This case is unique since no literature currently exists describing CTP-guided stenting in bilateral traumatic carotid artery dissection. It shows an early failure of medical management in controlling a progressive CAD, complicated by a large neurological burden caused by cerebral hypoperfusion. Often patients are on anticoagulation, which negates the use of IV thrombolysis. In our case, CTP highlighted the areas of ischaemic penumbra and the risk of bleeding into an already established infarct. Therefore, bilateral carotid stenting was safely utilised, suggesting that endovascular treatment is a good alternative to achieve reperfusion in areas of ischaemic penumbra caused by carotid artery dissection where anticoagulation or antiplatelets have failed.

\section{REFERENCES}

1. Leys D, Bandu L, Hénon H, Lucas C, Mounier-Vehier F, Rondepierre P, et al. Clinical outcome in 287 consecutive young adults (15 to 45 years) with ischemic stroke. Neurology 2002;59:26-33.

2. Redekop GJ. Extracranial carotid and vertebral artery dissection: a review. Can J Neurol Sci 2008;35:146-152.

3. Menon R, Kerry S, Norris JW, Markus HS. Treatment of cervical artery dissection: a systematic review and meta-analysis. J Neurol Neurosurg Psychiatry 2008;79:1122-7.

4. Engelter ST, Brandt T, Debette S, Caso V, Lichy C, Pezzini A, et al. Antiplatelets versus anticoagulation in cervical artery dissection. Stroke 2007;38:2605-11.

5. Kennedy F, Lanfranconi S, Hicks C, Reid J, Gompertz P, Price C, et al. Antiplatelets vs anticoagulation for dissection: CADISS nonrandomized arm and meta-analysis. Neurology 2012;79:686-9.

6. Lichy C, Metso A, Pezzini A, Leys D, Metso T, Lyrer P, et al. Predictors of delayed stroke in patients with cervical artery dissection. Int J Stroke 2012;10:1-4.

7. Droste DW, Junker K, Stögbauer F, Lowens S, Besselmann M, Braun B, et al. Clinically silent circulating microemboli in 20 patients with carotid or vertebral artery dissection. Cerebrovasc Dis 2001;12:181-5.

8. Cohen JE, Ben-Hur T, Rajz G, Umansky F, Gomori JM. Endovascular stent-assisted angioplasty in the management of traumatic internal carotid artery dissections. Stroke 2005;36:e45-7.

9. Xianjun H, Zhiming Z. A systematic review of endovascular management of internal carotid artery dissections. Interv Neurol;1:164-70.

10. Merckel LG, Van der Heijden J, Jongen LM, van Es HW, Prokop M, Waaijer A. Effect of stenting on cerebral CT perfusion in symptomatic and asymptomatic patients with carotid artery stenosis. AJNR Am J Neuroradiol 2012;33:280-5. 